\title{
Aplicabilidade de uma escala de risco para organização do processo de trabalho com famílias atendidas na Unidade Saúde da Família em Vitória (ES)
}

\author{
Applicability of the risk scale to the organization \\ of the work process with families in a health care unit \\ in the city of Vitória, Espírito Santo State
}

\author{
Fernanda Gomes do N ascimento ${ }^{1}$ \\ Thiago N ascimento do Prado ${ }^{2}$ \\ Heleticia Scabelo Galavote ${ }^{1}$ \\ PauleteAmbrósio $\mathrm{M}$ aciel $^{3}$ \\ Rita de Cássia Duarte Lima ${ }^{3}$ \\ Ethel Leonor Noia M aciel ${ }^{1}$
}

${ }^{1} \mathrm{Núcleos}$ deEstudosem Saúde Coletiva, Universidade Federal do Espírito Santo.

Av. M arechal Campos 1.468, M aruípe. 29040-091

Vitória ES. emaciel@ndi.ufes.br ${ }^{2}$ Laboratório de Epidemiologia do Núcleo deDoenças Infecciosas, Universidade Federal do Espírito Santo.

${ }^{3}$ Departamento de Enfermagem, Universidade Federal do Espírito Santo.
Abstract The objective of this study was to evaluate the applicability of Coelho's scale (CS) and to determineits adaptation to the reality observed in the families accompanied in the $M$ aria Rangel Passos health care unit (USFM RP) in the city of Vitória, Espírito Santo State. This is a cross-sectional exploratory, descriptivestudy developed in the area of health of Greater M aruípe. First, it was made the classification of the families according to CS and the results were presented for a discussion group that proposed changes and possiblealterationsin CS, being thenew tool denominated as "U FES criterium". In order to verify the applicability of that instrument and to accomplish a comparison with the obtained results of the classification for CS, thirty families assisted in USFM RP were randomly selected and visited, including by the HCA. This new scale allowed to identify a larger number of families of minimum risk and to guide the several actions accomplished daily in a health care unit, aiming to define priorities and to reorient the practices exercised by the professionals heading for the consolidation of a more even and integral attendance, centered in the service of the real social needs in health of the familiesassisted in theextent of the Family $\mathrm{H}$ ealth Strategy (FHS).

Key words Family Health Strategy, Coelho scale, Work process
Resumo 0 objetivo deste estudo foi avaliar a aplicabilidade da escala de escala de Coelho (EC) e determinar a sua adequação à realidade observada nas famíliasacompanhadas pela U nidadede Saúde M aria Rangel Passos (USFM RP) no município de Vitória (ES). Trata-se de um estudo exploratório-descritivo de corte transversal, desenvolvido na região de saúde da Grande M aruípe. A princípio, foi feita a classificação das famílias segundo a EC e os resultados foram apresentados a um grupo de discussão, que propôs mudanças e possíveis alterações na EC, sendo a nova ferramenta denominada como "critério UFES". Para verificar a aplicabilidade desse instrumento erealizar uma comparação com os resultados obtidos da classificação pela EC, foram selecionadas, de forma aleatória, e visitadas, inclusive pelo ACS, trinta famílias atendidas na U SFM RP. Esta nova escala permitiu identificar um maior número de famílias de risco mínimo e orientar as diversas ações que são realizadas no cotidiano de uma UnidadedeSaúde, com o objetivo de definir prioridades e reorientar as práticas exercidas pelos profissionais rumo à consolidação de uma assistência mais equânimeeintegral, centrada no atendimento das reais necessidades sociais em saúde dasfamílias atendidas no âmbito da Estratégia de Saúde da Família.

Palavras-chave Estratégia Saúdeda Família, Escala de Coelho, Processo de trabalho 
Introdução

A Constituição Federal de 1988, ao instituir o SistemaÚ nico deSaúde(SUS), define, por meio do seu artigo 196, um referencial paradigmático desaúde como direito de todos e dever do Estado, garantido mediante políticas sociais e econômicas.

Apesar do SUS representar, na história da saúde pública no Brasil, uma das principais inovações da reforma do Estado brasileiro, contrapondo-se ao modelo hegemônico estabelecido pelo Estado capitalista, ainda persistem desafios na implementação dos sistemas de saúde, dos quais destacamos, inicialmente, as questões relacionadas com os recursos humanos, perfis dos gestores públicos e os processos de monitoramento e avaliação que têm dado sustentação aos modelos de atenção no SUS ${ }^{1}$.

Dentre estes indicadores para monitorar e avaliar as ações executadas na atenção primária, destacam-seaqueles desenvolvidos no âmbito da Estratégia Saúde da Família (ESF).

Espera-sequeuma estratégia eficientee resolutiva seja capaz de resolver $80 \%$ da demanda dos serviços de saúde de uma comunidade, já que essa demanda concentra-se em poucos problemas, tornando-se peça central em todo o processo de reorganização das redes e sistemas de saúde?

Os profissionais de saúde, por sua vez, de vem apresentar capacidade técnica para identificar ehierarquizar as necessidades sociais em saúde, organizar saberes einstrumentos na configuração de modelos tecnológicos de intervenção e avaliar o impacto das intervenções realizadas. Além da capacidade técnica, devem agregar capacidade de negociação para a prática intersetorial e capacidade de fornecer evidências cientificamente embasadas para auxiliar a tarefa regulatória do Estado moderno no campo da saúde.

No entanto, sabe-seque, ainda hoje, um grande problema da ESF, ainda em construção no Brasil, se refere à demanda desordenada, que continua suprimindo a demanda organizada dentro das Unidades de Saúde da Família (USF). Dentro dessa problematização, torna-se evidente a necessidade de um instrumento que possi bilitepriorizar as visitas domiciliárias en quanto ação indispensável dentro do processo de trabalho das equipes multiprofissionais inseridas nas USF.

Com este propósito, foi elaborada uma escala de risco familiar baseada na ficha A do Sistema de Informação da Atenção Básica (SIAB), com a finalidade deestabelecer prioridades na visita domiciliária ${ }^{3}$. A "escala de Coelho" desponta, então, como uma ferramenta de avaliação e acompanhamento da realidade social eeconômicano contexto de vida de cada família, reconhecendo as reais necessidades de saúde no contexto da ESF.

0 presente estudo teve como objetivo avaliar a aplicabilidade da escala de risco familiar (escala de Coelho) e determinar a adequação deste instrumento à realidade observada nas famílias acompanhadas pela Unidade de Saúde da Família M aria Rangel Passos (USFM RP) no município de Vitória (ES).

\section{Metodologia}

Trata-se deum estudo exploratório-descritivo de cortetransversal, desenvolvido na região de saúdeda Grande M aruípe, no município de Vitória, na qual está inserida a Unidade de Saúde da Família M aria Rangel Passos (USFM RP). A escolha deste cenário se justifica por ser a região na qual - Centro de Ciências da Saúde da Universidade Federal do Espírito Santo se localiza e também o cenário de práticas dos acadêmicos dos cursos da área da saúde. A U SFM RP abrange os bairros Consolação, Jaburu, Gurijica, H orto, Floresta, São Benedito e contempla áreas, na maioria, de difícil acesso, por se tratar, em grande parte, de morro epor ter alto índice de criminalidade, atrelado a vários problemas sociais.

Essa Unidade de Saúde é composta, atualmente, por seis equipes de saúde, sendo quecada equipe é composta por cinco microáreas (exceto a equipe um, com seis microáreas ea equipetrês, que atualmente é composta por três microáreas), atendidas, cada uma, por um agente comunitário de saúde (ACS). Cada equipe também é composta por enfermeiro, técnico eauxiliares de enfermagem, médico, cirurgião-dentista e é assessorada pelo assistente social e psicólogo.

A coleta de dados foi desenvolvida no período de março a novembro de 2006. A pesquisa foi estruturada em quatro etapas. Na primeira, realizou-se a classificação das famílias cadastradas na USFM RP segundo a escala de Coelho ${ }^{3}$, categorizando-as em R1, R2 eR3, conformedemonstrado no processo de pontuação do risco familiar no Quadro 1. Esta etapa foi realizada a partir da análise das 2.150 fichas A do Sistema de Informação da atenção Básica (SIAB) preenchidas pelos ACS na primeira visita às famílias. Com isso, é possível a ESF reconhecer os indicadores demográficos, socioeconômicos e nosológicos referidos nas famílias da área de abrangência do ACS. Também foram realizadas visitas domiciliá- 
Quadro 1. Dados da ficha A do SIAB e escore de pontuação de risco/Classificação das famílias segundo pontuação.

\begin{tabular}{|l|l|l|}
\hline \multicolumn{1}{|c|}{ Dados da ficha A } & & Escore \\
\hline Acamado & & 3 \\
\hline Deficiência física & & 3 \\
\hline Deficiência mental & & 3 \\
\hline Baixas condições de saneamento & 3 \\
\hline Desnutrição (grave) & & 2 \\
\hline Drogadição & & 2 \\
\hline Desemprego & & 1 \\
\hline Analfabetismo & & 1 \\
\hline M enor de seis meses & & 1 \\
\hline M aior de setenta anos & & 1 \\
\hline Hipertensão arterial sistêmica & Se maior que 1 & 3 \\
\hline Relação morador/cômodo & Se igual a 1 & 2 \\
\hline & Se menor que 1 & 0 \\
\hline \multicolumn{1}{|c|}{ Escore total } & Classificação de risco & \\
\hline & R1 & \\
\hline Escore 5 ou 6 & R2 & \\
\hline Escore 7 ou 8 & R3 & \\
\hline M aior que 9 & & \\
\hline
\end{tabular}

Fonte: Escala de Coelho ${ }^{3}$.

rias para observar a classificação das famílias de acordo com esta escala.

A segunda etapa contemplou a apresentação dos resultados obtidos da classificação das famílias para um grupo de discussão da escala composta por todos os profissionais inseridos na unidade.

De posse dos resultados desta discussão, propusemos uma modificação na escala de Coelho e esta nova ferramenta foi denominada "critério UFES". Na terceira etapa, foram realizadas visitas domiciliárias a algumas famílias, visando à adequação dessa nova ferramenta à realidade observada nas famílias atendidas pela USFM RP.

Foram selecionadas, de forma aleatória, e visitadas, inclusive pelo ACS, trinta famílias atendidas na USFM RP, o que permitiu verificar a aplicabilidade e a implementação do instrumento denominado "critério UFES" e comparar com os resultados obtidos da classificação pela escala de Coelho.

Toda a informação obtida durante a coleta de dados deu origem a um banco de dados que foi armazenado no software M icrosoft Excel. Em seguida, foram transferidos para o programa estatístico STATA 9.0 para cálculos de frequência absoluta e relativa. 0 estudo foi aprovado pelo Comitê de Ética em Pesquisa com Seres Humanos do Centro de Ciências da Saúde da Universidade Federal do Espírito Santo (UFES) e pelo ComitêdeÉtica da Secretaria M unicipal deSaúde de Vitória.

\section{Resultadosediscussão}

Foram analisadas 2.150 fichas A do SIAB, correspondendo à quantidade de famílias cadastradas pelos Agentes Comunitários de saúde (ACS) na USFM RP. Dessas, a partir da aplicação da escala de Coelho, foram identificadas 1.192 (55,44\%) famílias sob risco, que apresentavam escore total mínimo de 5 . Foram categorizadas 956 (89\%) famílias como R1, 150 (7\%) famílias (R2) e 86 (4\%) famílias como R3 (Tabela 1).

Ao analisarmos estes números, verificamos que a maior parte das famílias, cerca de $55,44 \%$, atendidas pela USFM RP está sob risco familiar. Além disso, foi verificada uma quantidade significante de famílias consideradas como de risco máximo 3 eque necessitam de uma maior atenção por parte dos profissionais de saúde, o que 
demanda o maior número de visitas domiciliárias e serviços de saúde.

Quando analisamos a distribuição das famílias categorizadas como R1, R2 e R3 na escala de Coelho por equipe de saúde (Quadro 2), observamos que, em algumas equipes, o número de famílias R3 (que nos critérios da escala deveriam ser prioridade na definição do maior número de visitas domiciliárias) é percentualmente maior, como as equipes II (8), III (9) e VI (7), em comparação com as equipes I (2), IV (1), eV (2), ou seja, as equipes possuem diferentes avaliações de risco conforme a ótica de análise sistemática de cada profissional. Há, portanto, uma necessidade de maiores investimentos nos instrumentos de avaliação e análise das ações, assim como 0 estabelecimento de estratégias para priorizá-las, visando semprea consolidação do diálogo entre os diferentes profissionais da equipe, com o objetivo de criar um espaço de debates e definição de prioridades no que se refere ao atendimento das reais necessidades de saúde dos indivíduos e seu coletivo. É evidenteque, dentro deuma mesma equipe, diferentes microáreas podem possuir avaliações de risco divergentes, podendo-se, a partir daí, estabelecer estratégias para priorizar visitas domiciliárias naquelas regiões de maior necessidade. Além disso, o investimento de recursos, tanto humanos quanto financeiros, pode seguir a mesma estratégia3.

Tabela 1. Dados da ficha A do SIAB e escore de pontuação de risco/Classificação das famílias segundo pontuação.

\begin{tabular}{|c|c|c|c|c|c|c|}
\hline $\begin{array}{c}\text { Classificação } \\
\text { de risco }\end{array}$ & $\begin{array}{c}\text { Equipe I } \\
n=120 \\
n(\%)\end{array}$ & $\begin{array}{c}\text { Equipe II } \\
n=207 \\
n(\%)\end{array}$ & $\begin{array}{c}\text { Equipe III } \\
n=208 \\
n(\%)\end{array}$ & $\begin{array}{c}\text { Equipe IV } \\
n=195 \\
n(\%)\end{array}$ & $\begin{array}{c}\text { Equipe V } \\
n=195 \\
n(\%)\end{array}$ & $\begin{array}{c}\text { Equipe VI } \\
n=197 \\
n(\%)\end{array}$ \\
\hline $\mathrm{R} 1$ & 170 (94) & $149(82)$ & 147 (81) & $165(91)$ & 165 (91) & $160(88)$ \\
\hline $\mathrm{R} 2$ & $14(4)$ & 34 (10) & 34 (10) & 27 (10) & $24(7)$ & $17(5)$ \\
\hline R3 & $6(2)$ & 24 (8) & $27(9)$ & $3(1)$ & $6(2)$ & $20(7)$ \\
\hline
\end{tabular}

Quadro 2. Dados da ficha A do SIAB e escore de pontuação de risco de acordo com o "critério UFES"/ Classificação das famílias segundo pontuação obtida.

\begin{tabular}{|l|c|c|}
\hline \multicolumn{1}{|c|}{ Dados da ficha A } & & Escore \\
\hline Acamado & & 3 \\
\hline Deficiência física & & 3 \\
\hline Deficiência mental & & 3 \\
\hline Baixas condições de saneamento e/ou higiene & & 3 \\
\hline Risco para desnutrição >P10 & & 2 \\
\hline Uso de drogas lícitas e ilícitas & & 2 \\
\hline Desemprego & & 2 \\
\hline Doença crônica & & 2 \\
\hline Violência familiar & & 1 \\
\hline Analfabetismo & & 1 \\
\hline Menor de um ano & Se maior que 1 \\
\hline Maior de setenta anos & Se igual a 1 & 3 \\
\hline Relação morador/cômodo & Se menor que 1 & 2 \\
\hline & Classificação de risco & \\
\hline & R1 & \\
\hline Escore total & R2 & \\
\hline Escore $\geq 1$ & R3 & \\
\hline Escore 7 a 8 & & \\
\hline Escore maior que 9 & & \\
\hline
\end{tabular}


Após o levantamento de dados das fichas $A$ do SIAB, foi feito, juntamente com um ACS, a análise da "escala de Coelho" comparada com a realidade das famílias cadastradas na USFM RP. Em todas as famílias R3 desta equipe, foi possível a realização das visitas domiciliárias, tornando mais evidentes alguns dados já encontrados na análise com o ACS.

$\mathrm{N}$ as visitas realizadas, tornou-se evidente 0 fato de que essas famílias R3 realmente estavam sob risco máximo, segundo a escala de Coelho. Algumas características relacionadas à organização, inerente à organização familiar, foram similares nas avaliações, destacando-se o desemprego, o uso de drogas e precárias condições de habitação e saneamento básico, diferenciando-as das famílias classificadas como R1. Esse fato foi constatado por Coelho3, ao avaliar o que deve ser pontuado e individualizado de acordo com a abordagem à comunidade.

0 autor ressalta que, em uma comunidade onde não há saneamento básico, a designação, nesse caso, será de "área de risco" e não de "risco familiar", já que envolve aspectos que perpassam o espaço domiciliar das famílias, estando relacionado à própria construção e promoção de políticas públicas saudáveis que se expressam através de diversas abordagens complementares, que incluem legislação, medidas fiscais, taxações e mudanças organizacionais e por ações coordenadas, que apontam para a equidade em saúde, distribuição mais equitativa da renda e políticas sociais4. N esse parâmetro, o pesquisador teveseu objetivo alcançado através de nossa pesquisa, já que as famílias visitadas condizem com o perfil de risco proposto por sua escala.

Observamos, durante as visitas domiciliárias, que alguns critérios não haviam sido classificados na escala de risco de Coelho, como as condições dehigiene do ambiente, ditos relevantes pela equipe. Além disso, Prietsch 5 aponta queo principal fator de risco ambiental para o desenvolvimento de doenças do trato respiratório inferior constitui na exposição a agentes poluidores domésticos. Ressalta, ainda, que a presença de alérgenos inalados, especialmente o ácaro do pó doméstico, componenteda saliva epêlos deanimais, também está relacionada ao incremento de resposta inflamatória da mucosa respiratória.

Uma escala de risco deve sempre se materializar na constatação de que o risco é "proteiforme", ou seja, envolve diferentes aspectos, dentreeles os econômicos (desemprego, miséria), ambientais (poluição), de condutas pessoais (alimentação, atividadefísica), dimensões interpessoais (sexua- lidade) e criminais (eventos vinculados à violência urbana) ${ }^{6}$. Todos os riscos citados se apresentam em meio a uma teia de relações e inter-relações, extravasando para o âmbito sociocultural, assumindo aspectos de signos e símbolos experenciados nas vivências dos serviços de saúde no espaço de construção das relações de grupos sociais. N esse sentido, a abordagem do risco familiar deve sempre ser centrada nas múltiplas relações da casualidade e determinação social.

O bservamos, nas visitas, que as condições de higieneeram precárias, havia o convívio direto de indivíduos com animais domésticos, moradias com pouca ventilação e grande quantidade de ácaros (observados pela inadequada higienização do ambiente). Assim sendo, a questão da higiene nos espaços do domicílio perpassa os aspectos relacionados somenteao ambiente externo, como o saneamento básico, estando relacionada a aspectos culturais e comportamentais que se referem à própria constituição das relações sociais, econômicas e de poderes estabelecidos numa sociedade de desigualdades e iniquidades.

Outro ponto a ser destacado está relacionado às famílias nas quais há apenas um residente, não acamado, não hipertenso, não diabético, com boas condições de moradia, mas desempregado, usuário de drogas, etilista, o que, segundo os nossos critérios, seriam componentes de famílias de risco considerável (R3). No entanto, de acordo com a "escala de Coelho", são famílias de risco mínimo.

M artin7, sob este aspecto, ao se deter à situação de risco, aponta que a situação de risco pessoal e social de uma família é entendida como a situação vivida pela família em desvantagem, que Ihe determina características gerais de múltiplos problemas. Esta denominação não pretende ser um julgamento de valor, mas descreve algumas características que são gerais nesta situação. Em geral, estas famílias possuem educação e recursos monetários limitados, o que as incapacita defuncionar adequadamentena sociedade mais ampla. 0 autor ressalta, ainda, que tais famílias possuem características relativas à estrutura e papéis familiares, processos de comunicação familiar e socialização das crianças, que são peculiares e determinantes do risco, tanto pessoal como social.

Castiel ${ }^{6}$ ressal ta que, recentemente, produziuse um acentuado deslocamento das intervenções curativasterapêuticas paraum gerenciamento das populações derisco, o que pode gerar tanto ações inócuas quanto benéficas para nossa saúde, como modos de exclusão e controle. Assim, quando o olhar do profissional privilegia as populações con- 
sideradas como de risco, pode desprezar as necessidades de saúde dos considerados "normais" ou "fora de risco", o que é evidente nas avaliações de escal as de risco nos grupos sociais.

Esses foram apenas alguns exemplos de famílias que não foram alcançadas pela "escala de Coelho". 0 problema, portanto, não está entre as famílias que foram classificadas como R3, mas entre as que não o foram. Com isso, a partir do estudo realizado com a escala de Coelho e comparado com a realidade dos bairros atendidos pela USFM RP e as visitas domiciliárias feitas com os ACS, surgiu a necessidade de elaborar uma nova escala, denominada "critério UFES", conforme demonstrada na Tabela 2. Essa tem como objetivo principal tentar reclassificar as famílias da USFM RP o mais próximo de suas realidadese adaptá-las às reais situações que colocam em risco uma família atendida pela unidade.

Dados como "acamado", "deficiência física e mental", "desemprego", "analfabetismo", "maior que setenta anos" e "relação morador/cômodo" foram mantidos, visto que são de extrema importância para se classificar uma família com risco. Dentre os que foram modificados estão "cobertura do saneamento", "desnutrição (grave)", "drogadição", "menor de seis meses", "hipertensão arterial sistólica" e "diabetes mellitus".

No dado "condições de saneamento", incluiuse, também, "baixas condições dehigiene". A "desnutrição (grave)" passou a ser classificada como "risco para desnutrição < P10", com base nos estudos de Soares ${ }^{8}$, que atribui desnutrição às crianças com medidas abaixo do percentil $10 \mathrm{ea}$ normalidade com medidas entre os percentis 10 e 90. A visita domiciliar deve servir com a finalidade de prevenir qualquer agravo; portanto, foi considerado que se deve priorizar a visita domiciliária antes que a desnutrição alcance seu nível mais grave (percentil 3).

Em relação à "drogadição", foi modificada a classificação para "uso de drogas lícitas e ilícitas", visto que o etilismo e o tabagismo são fatores de risco dentro de um ambiente familiar e não somente o uso de drogas ilícitas. Segundo Sabry ${ }^{9}$, 0 tabagismo e o etilismo têm sido relacionadoscom a prevalência de várias doenças ou distúrbios. 0 mesmo autor afirma que o tabagismo é responsável por $1 / 5$ das mortes por doenças cardíacas. Ressalta ainda que o hábito de fumar vem sendo relacionado com maior prevalência de hipertensão arterial, bem como está associado ao desencadeamento de doenças pulmonares.
Quanto ao dado "menor de seis meses", citado na escala de Coelho", foi alterado para "menor de um ano", no "critério UFES", pois segundo Campos $^{10}$, a taxa de mortalidade infantil (TMI) éreconhecida não só como indicador das condições de saúde da população menor de um ano, mas também como a variável que melhor reflete as condições gerais de vida, sendo considerada síntese da qualidade de vida e do nível de desenvolvimento de uma população .

Em relação aos dados "hipertensão arterial sistólica" e "diabetes mellitus", referenciados na escala de Coelho", foram englobados em "doenças crônicas", pois, de acordo com o perfil epidemiológico das famílias atendidas na USFM RP, havia outras doenças consideradas de risco, como bronquiteque, na escala de Coelho, não éclassificada como tal. A importância das doenças crônicas não transmissíveis (DCNT), no perfil atual de saúde das populações, é extremamente relevante. Estimativas da Organização Mundial de Saúde (OMS) apontam que as DCNT já são responsáveis por $58,5 \%$ de todas as mortes e por $45,9 \%$ da carga total global de doenças, expressas por anos perdidos de vida saudável ${ }^{11}$. Foi incorporado, também, na escala "critério UFES", o dado violência familiar, visto que essa se tornou uma problemática constante observada nas famílias atendidas pela USFM RP, além de sua considerada relevância no campo da Vigilância da Saúde, conforme referido por autores como Soares ${ }^{12}$.

Depois de realizada a classificação dos critérios, foram colocados os escores devidos (Tabela 2). A classificação das famílias em R1, R2 eR3 mantiveram-se. Contudo, a pontuação exigida, ou seja, o escore total necessário para a classificação de uma família em risco 1, foi alterado. Enquanto na escala de Coelho era necessária a obtenção de um escore total de 5 para considerar uma família de risco mínimo, na escala "critério UFES" a obtenção de um escore maior/igual a 1 passa a classificar a família como de risco mínimo.

Esta reclassificação, e alteração, da escala de Coelho, permitiram identificar um maior número defamílias derisco mínimo, as quais precisarão da atenção especial dos profissionais de saúde da USFM RP. Essas serão alvos dos programas de promoção à saúde e prevenção de agravos ofertados pela unidade. Estas atuações evitarão que as famílias derisco mínimo venham a se tornar de risco máximo (R3) (Tabela 2). 
Tabela 2. Comparação dos escores obtidos das 30 famílias da equipe 1 em relação à "escala de Coelho" e à escala "critério UFES".

\begin{tabular}{|c|c|c|}
\hline Família & Escala de Coelho & Critério UFES \\
\hline 1 & $9(\mathrm{R} 3)$ & $15(\mathrm{R} 3)$ \\
\hline 2 & $8(\mathrm{R} 2)$ & $9(\mathrm{R} 3)$ \\
\hline 3 & $7(\mathrm{R} 2)$ & $9(\mathrm{R} 3)$ \\
\hline 4 & 0 & $4(\mathrm{R} 1)$ \\
\hline 5 & $8(\mathrm{R} 2)$ & 10 (R3) \\
\hline 6 & 4 & $7(\mathrm{R} 2)$ \\
\hline 7 & 4 & $7(\mathrm{R} 2)$ \\
\hline 8 & 7 (R2) & 11 (R3) \\
\hline 9 & 4 & $7(\mathrm{R} 2)$ \\
\hline 10 & 3 & $4(\mathrm{R} 1)$ \\
\hline 11 & $5(\mathrm{R} 1)$ & $6(\mathrm{R} 1)$ \\
\hline 12 & 7 (R2) & $7(\mathrm{R} 2)$ \\
\hline 13 & 4 & $6(\mathrm{R} 1)$ \\
\hline 14 & $6(\mathrm{R} 1)$ & $7(\mathrm{R} 2)$ \\
\hline 15 & 2 & $6(\mathrm{R} 1)$ \\
\hline 16 & $8(\mathrm{R} 2)$ & $8(\mathrm{R} 2)$ \\
\hline 17 & 4 & $5(\mathrm{R} 1)$ \\
\hline 18 & 3 & $6(\mathrm{R} 1)$ \\
\hline 19 & $6(\mathrm{R} 1)$ & $6(\mathrm{R} 1)$ \\
\hline 20 & $5(\mathrm{R} 1)$ & $5(\mathrm{R} 1)$ \\
\hline 21 & 3 & $6(\mathrm{R} 1)$ \\
\hline 22 & 0 & $4(\mathrm{R} 1)$ \\
\hline 23 & $5(\mathrm{R} 1)$ & $6(\mathrm{R} 1)$ \\
\hline 24 & 0 & 0 \\
\hline 25 & 3 & $5(\mathrm{R} 1)$ \\
\hline 26 & 2 & $2(\mathrm{R} 1)$ \\
\hline 27 & 0 & 0 \\
\hline 28 & 2 & $4(\mathrm{R} 1)$ \\
\hline \multirow[t]{2}{*}{29} & $5(\mathrm{R} 1)$ & $6(\mathrm{R} 1)$ \\
\hline & 2 & $4(\mathrm{R} 1)$ \\
\hline
\end{tabular}

\section{Consideraçõesfinais}

A implementação da escala "critério UFES" surgiu do diálogo entre todos os profissionais inseridos na USFMRP e a partir da constatação de que a escala de Coelho não contemplava a realidade das famílias atendidas pelo serviço. A meta não era a identificação de famílias que estariam sob risco e que precisariam de uma maior atenção por parte dos serviços, mas sim a prevenção e a promoção da saúde.

Compreendemos a importância do enfoque da promoção da saúde na realidade vivenciada no cenário estudado. Ressaltamos a importância da busca por uma compreensão ampla do processo saúde-doença e de seus determinantes, inseridos no campo da saúde, estimulando a articulação equipe de saúdee comunidade.
Consideramosa necessidade de quehaja profissionais com capacidade de escuta ampliada às necessi dades sociais, reconhecen do a construção de um projeto terapêutico singular fundamentado no acolhimento, e não somente trabalhadores classificadores de risco, mas, sobretudo, responsáveis pela saúde individual e coletiva ${ }^{13}$.

0 que propomos são mudanças e adaptações dos instrumentos de avaliação de risco de acordo com a realidade de cada serviço e da comunidadereferida, compreenden do quemais do que identificar uma família como sendo de risco 1 ou 3, é saber determinar os fatores inter-relacionados ao contexto de vida de cada indivíduo, buscando a gênese dos determinantes dos processos saúde- doença, a fenomenologia dos fatores relacionados ao modo de vida de cada família, tendo semprea ideia de que as desi gualdades e iniquidades envolvem aspectos que estão muito além das portas ou janelas dos domicílios.

Nesse sentido, somos adeptos das proposições deAyres ${ }^{14}$, queaponta avulnerabilidadecomo conceito estruturante das abordagens de avaliação das condições de vida e saúde, já que está fundamentada na copresença, mutualidade, interferência, relatividadeena inconstância. No entanto, instrumentos que se proponham a avaliar a vulnerabilidade não são ainda muito difundidos, estando o atual enfoque no risco e seu caráter meramente probabilístico. Assim, a busca de novas análises, mais condizentes com a realidade particular de cada cenário, perpassa pela necessidade de reorientarmos os instrumentos de acompanhamento e diagnóstico situacional de acordo com as características de cada contexto, sempre atentando para o fato de que, sem uma compreensão clara do que consideramos como risco e quais são os seus determinantes, não teremos possibilidade de apreender os reais fatores que se interpõem nos espaços de vida das famílias.

0 "critério UFES" representa uma reorientação da escala de Coel ho no sentido de se adaptar ao contexto social e buscar redefinir a classificação das famílias segundo o enfoque de risco, reconhecendo a possibilidade de que, com novos estudos nesse contexto de práticas, poderemos, então, redirecionar a escala de risco para a de vulnerabilidade, com o objetivo de avaliar as suscetibilidades populacionais, analisar a resposta social e os determinantes de vida e saúde dos indivíduos, evitando a imposição de paradigmas comportamentalistas e construindo um paradigma construtivista, para que as pessoas possam de fato se mobilizar e achar alternativas práticas que permitam o enfrentamento das situações que 
as vulnerabilizam sem, contudo, retirar do Estado e do setor saúde a responsabilidade pela proposição e execução de políticas públicas condizentes com as reais necessidades de saúde construídas e reconstruídas nos cenários de vida dos grupos sociais em nosso país.

\section{Colaboradores}

FG Nascimento participou da elaboração do tema, construção do quadro teórico, coleta dos dados, análise e delineamento final do estudo. TN Prado e HS Galavote participaram da coleta dos dados e revisão final. PA M aciel eRCD Lima participaram da elaboração do tema e revisão final. ELN Maciel participou da elaboração do tema, construção do quadro teórico, análise e revisão final.

\section{Referências}

1. Levcovitz E, Machado CV, Lima LDA. As políticas de saúde nos anos 90: relações intergovernamentais e 0 papel das normas operacionais básicas. Cien Saude Colet 2002; 6(2):269-291.

2. Aleixo JLM. Atenção Primária à Saúde e o Programa de Saúde da Família: perspectivas de desenvolvimento no início do terceiro milênio. Revista M ineira de Saúde Pública 2002; 1(1):1-16.

3. Coelho FLG. Visita domiciliar [palestra]. In: I Congresso M ineiro de M edicina de Família e Comunidade: Qualidade e Transformação; 2003; Belo Horizonte.

4. Buss PM. Uma introdução ao conceito de promoção da Saúde. In: Czeresnia D, Freitas CM , organizadores. Promoção da Saúde: conceitos, reflexões e tendências. Rio de Janeiro: Fiocruz; 2004. p. 15-37.

5. Prietsch SO, Fischer GB, Cesar JA, Lempek BS, Barbosa LV Jr, Zogbi L, Cardoso OC, Santos AM. Doença respiratória em menores de 5 anos no sul do Brasil: influência do ambiente doméstico. Rev. Panam. Salud Públ. 2003; 13(5):303-310.

6. Castiel LD. Vivendo entre exposições e agravos: A teoria da relatividade do risco. Hist. cienc. saude$M$ anguinhos 1996; 3(2):237-264.

7. Martin VB, Angelo M. Significado do conceito saúde na perspectiva de famílias em situação de risco pessoal e social. Rev. Latino-am Enfermagem 1998; 6(5):45-51.

8. Soares NT, Parente WG. Desnutrição e resultados de reabilitação em Fortaleza. Rev. Nutr. 2001; 14(2):103-110.

9. Sabry MOD, Sampaio HAC, Silva M GC. Tabagismo e etilismo em funcionários da Universidade Estadual do Ceará. J Pneumol. 1999; 25(6):313-320.
10. Campos TP, Carvalho MS, Barcellos CC. Mortalidade infantil no Rio de Janeiro, Brasil: Áreas de risco e trajetória dos pacientes até os serviços de saúde. Rev. Panam. Salud Públ. 2000; 8(3):164-171.

11. Monteiro CA, Moura EC, Jaime PC, Lucca A, Florindo AA, Figueiredo ICR, Bernal R, Silva NN. $M$ onitoramento de fatores de risco para doenças crônicas por entrevistas telefônicas. Rev. Saude Publica 2005; 39(1):47-57.

12. Soares BM. Mulheres invisíveis: violência familiar e formações subjetivas [tese]. Rio de Janeiro (RJ): Instituto Universitário de Pesquisas do Rio de Janeiro; 1997.

13. Carvalho YM, Ceccim RB. Formação e Educação em Saúde: aprendizados com a Saúde Coletiva. In: Campos GW, M inayo M CS, Akermann M, Drumond M, Carvalho YM, organizadores. Tratado de Saúde Coletiva. São Paulo: Hucitec; 2006. p. 149-182.

14. Ayres JRCM, França JI, Calazans GJ, Saletti HC. O conceito de vulnerabilidade e as práticas de saúde: novas perspectivas e desafios. In: Czeresnia D, Freitas CM, organizadores. Promoção da saúde: conceitos, reflexões, tendências. Rio de Janeiro: Fiocruz; 2003. p.117-139.

Artigo apresentado em 19/12/2007

Aprovado em 27/06/2008

Versão final apresentada em 21/07/2008 